\title{
Interferometric imaging tests for the Large Binocular Telescope ${ }^{\star}$
}

\author{
S. Correia and A. Richichi \\ Osservatorio Astrofisico di Arcetri, Largo E. Fermi 5, I-50125 Firenze, Italy
}

Received July 5; accepted August 15, 1999

\begin{abstract}
We present an experiment of interferometric imaging for the Large Binocular Telescope (LBT), conducted at the $1.5 \mathrm{~m}$ TIRGO infrared telescope. The raw data were produced by simulating the LBT pupil with a mask on the secondary mirror. Two different conditions of $D / r_{0}$, where $D$ is the single aperture diameter and $r_{0}$ the Fried parameter, were simulated by the choice of $D$; field rotation was simulated by rotating the mask. The data set collected consists of several sequences of short exposure interferograms of one pointlike and one binary star in the $J$-band, for two different $D / r_{0}$ conditions. We show preliminary results, in particular concerning the fringe contrast loss with integration time. The ability of a Lucy-Richardson-based deconvolution algorithm to reconstruct an object from a set of LBT-like interferometric real data was demonstrated on the binary star $\gamma$ Leo. The retrieved binary parameter values are compared to catalog values, and a good astrometric agreement is found. Photometric and resolution limitations are also discussed. In a first part, the reconstruction method and preliminary numerical simulations of LBT image restoration using this method are presented.
\end{abstract}

Key words: instrumentation: interferometers methods: data analysis — techniques: image processing — techniques: interferometric telescopes — stars: imaging

\section{Introduction}

The Large Binocular Telescope (LBT) will consist of two $8.4 \mathrm{~m}$ mirrors on a common mount. When the two mirrors are coherently cophased, this will work as a total baseline

Send offprint requests to: S. Correia

e-mail: correia@arcetri.astro.it

* Based on observations collected at TIRGO (Gornergrat, Switzerland). TIRGO is operated by CNR-CAISMI, Arcetri, Italy. of $22.8 \mathrm{~m}$. In several respects, observations with the LBT in this configuration will differ from those with more "conventional" interferometers. For example, LBT will offer a large field of view, and will permit true imaging by simultaneously measuring all the Fourier components (Angel et al. 1998).

At the same time, the peculiar point spread function (PSF) and its rotation in the sky due to the alt-azimuthal mount, will require specific data acquisition algorithms, and specialized treatment in the data reduction process. With these points in mind, we started a project to perform tests and develop relevant software. The aim was to investigate the process of image formation and reconstruction at the LBT, taking into account the characteristics of the atmosphere, the telescope performance and adding realistic estimates for the detector read-out noise (RON).

Previously, at least two others groups of authors have already discussed LBT image reconstruction. However, only numerical simulations, based on different reconstruction techniques, have been carried out up to date. Reinheimer et al. (1997) have applied the so-called iterative building block method (bispectral analysis) to some point-like and extended objects. They presented a reconstruction method to apply to a speckle utilisation of LBT, with the known limitation in sensitivity of this observation mode. They complemented their simulations with a laboratory experiment. However, this latter was carried out under very favorable turbulence conditions $\left(r_{0}=2 \mathrm{~m}\right)$ and unspecified brightness of the source. Prior to this Hege et al. (1995) had explored the use of iterative blind deconvolution (IBD algorithm of Jefferies \& Christou 1993), on simulated LBT images of an extended object.

On the contrary, our work is based on real LBT-like data. In Sect. 2 we present the algorithm of reconstruction that we have used and modified. It is based on the Lucy-Richardson deconvolution algorithm (Richardson 1972; Lucy 1974), widely used in standard image restoration methods. Section 3 shows the potential of this 
reconstruction technique on some preliminary tests performed on simulated point-like and extended objects.

In Sect. 4 we present the experiment realized at the TIRGO observatory. The measurements allowed us to study some quantitative aspects concerning the process of image formation under low-order atmospheric degradation conditions (see Sect.4.1). Section 4.2 shows the result of the application of the reconstruction method on LBTlike data from the TIRGO experiment.

\section{The reconstruction method}

In this work, we used a Lucy-Richardson (LR) iterative algorithm adapted for multiple deconvolution, i.e. an algorithm based on the LR method that allows to retrieve full angular resolution images from LBT interferograms. This adaptation was first developed and tested at the University of Genova (Bertero \& Boccacci, in preparation) on simulated data made of one frame per orientation angle.

From this code, we have implemented the possibility to add the information of more than one frame per orientation angle, i.e. to do a simultaneous deconvolution of a set of frames per orientation angle. This approach is useful when dealing with noisy and atmospheric-degraded data, in the case of the LBT-like data we have collected. The method yields the common maximum-likelihood estimate object from the full data set. Details of this algorithm are outlined hereafter.

Let's denote $f(x, y)$ the brightness distribution of the object, and $h_{i}(x, y)$ the PSF corresponding to the $i^{\text {th }}$ baseline position angle of the LBT interferometer. For each position angle, we observe the interferogram intensity $g_{i}(x, y)$ defined by the object image convolution relationship:

$g_{i}(x, y)=h_{i}(x, y) * f(x, y)+n(x, y)$

where $n(x, y)$ refers to a spatially variant noise process. The original LR algorithm is a non-linear algorithm derived from Bayesian considerations, and based on the knowledge of the PSF. The principle consist in multiplying the result of each iteration by a correction factor $C^{k}(x, y)$, with $k$ denoting the iteration number, that relates to the remaining fitting error. In standard LR image restoration, i.e. for $i=1$, the iterative relation is the following:

$$
f^{k+1}(x, y)=f^{k}(x, y) C^{k}(x, y)
$$

with

$C^{k}(x, y)=h^{*}(-x,-y) *\left[\frac{g(x, y)}{h(x, y) * f^{k}(x, y)}\right]$

where $h^{*}(-x,-y)$ is the conjugate of $h(-x,-y)$. With a positive constant estimate $f^{0}(x, y)$, this algorithm leads to successive estimates $f^{k}(x, y)$ which are implicitly positive. Since different values of this constant do not lead to significant consequences in term of convergence rate, $f^{0}(x, y)$ was fixed to unity. Another interesting characteristic of this algorithm is the conservation of the total energy. It is also demonstrated (Shepp \& Vardi 1982) that this relation leads to the maximum-likelihood object estimate, under the assumption of a Poisson process in image formation.

In the multiple deconvolution case, i.e. for $i>1$, we simply sum the contribution of each position angle at each iteration. The iterative scheme is identical to Eq. (2) only the correction factor is changed into:

$C^{k}(x, y)=\frac{1}{H} \sum_{i=1}^{N}\left[h_{i}^{*}(-x,-y) * \frac{g_{i}(x, y)}{h_{i}(x, y) * f^{k}(x, y)}\right]$

where $N$ is the number of baseline position angles. $1 / H$ is a normalized term where

$H=\sum_{i=1}^{N}\left[\sum_{x, y} h_{i}(x, y)\right]$.

Because of the normalization of each $h_{i}(x, y)$ to unity, $H=N$.

The same approach is used when a set of frames per orientation angle $g_{i, j}(x, y)$ exists, with $j$ denoting the position of the frame in the set. Adaptation of the algorithm to this leaves unchanged Eq. (2) and only modifies the correction factor as following:

$C^{k}(x, y)=\frac{1}{H} \sum_{i=1}^{N} \sum_{j=1}^{M}\left[h_{i}^{*}(-x,-y) * \frac{g_{i, j}(x, y)}{h_{i}(x, y) * f^{k}(x, y)}\right]$

where $M$ is the number of frames per angle in the set. Note that the PSF may also be different, and therefore written as $h_{i, j}(x, y)$. But, concerning our data, we were not able to obtain a good knowledge of the corresponding PSF for each frame and therefore preferred a common PSF estimate.

\section{Image simulation and reconstruction test}

In this section, we present the application of the reconstruction method of Sect. 2 to numerical simulations of LBT imaging, taking into account the telescope performance and adding realistic estimates for the detector. In a first step, the construction of simulated interferometric observations was carried out using an ideally AO corrected PSF within the whole field of view. In addition we considered perfect optics and co-phasing of the two pupils. The PSF's were therefore modeled as cosine-modulated Airy functions. In the near future we plan to obtain a more realistic PSF modeling by taking into account the attainable level of AO correction. The simulated interferometric observations, performed in $R$-band, were obtained as the convolution of the target with the 


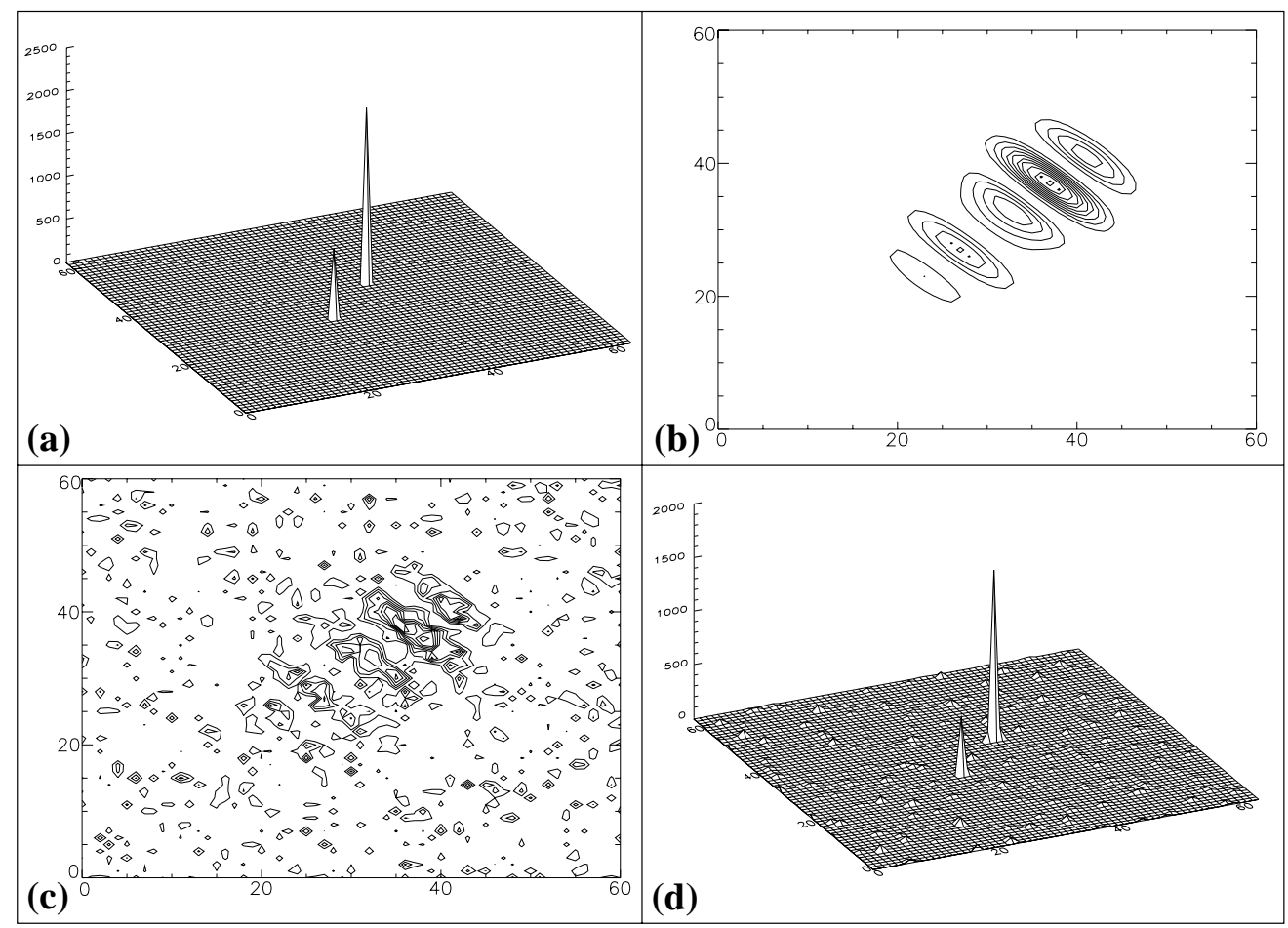

Fig. 1. Numerical simulation of interferometric image synthesis with LBT at optical wavelength of a binary star object. a) simulated binary star image with main component magnitude $m_{R}=29$ and $\Delta m_{R}=1$. b) one of the noise-free simulated interferograms of the binary (parallactic angle $\left.=45^{\circ}\right)$. c) same interferogram noise-contaminated (10 linear contours levels are shown). d) reconstructed binary star after 1000 iterations of the algorithm (the $z$-scale is in electrons)

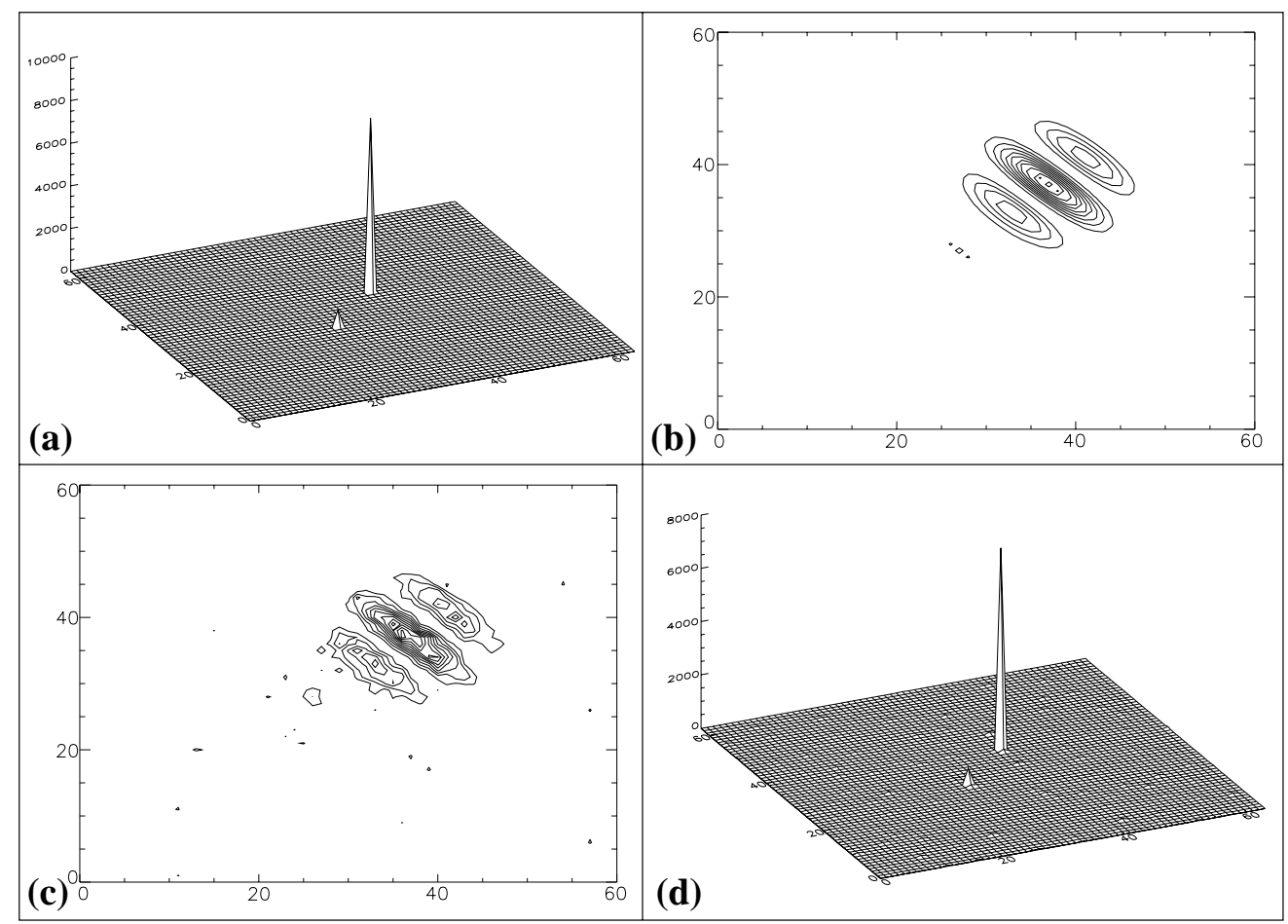

Fig. 2. Same as Fig. 1 for a binary star with main component magnitude $m_{R}=27.5$ and $\Delta m_{R}=2.5$ 
PSF corresponding to each parallactic angle, adding sky-background emission. Independent Poisson noise realizations were then computed, and realistic detector RON added for each parallactic angle. In all simulations presented below, we assumed $1000 \mathrm{~s}$ integration time per parallactic angle, $30 \%$ efficiency (mirrors + optics + detector), a sky brightness of $20.80 \mathrm{mag} / \operatorname{arcsec}^{2}$ and a RON equivalent magnitude of $35.8 \mathrm{mag} / \mathrm{pix}$.

Since the large extension ( $\sim 30^{\prime \prime}$ in $R$-band) of the foreseen AO corrected LBT field, it will be theoretically possible to obtain a sufficiently bright reference star in the field for PSF calibration. Indeed the average density of stars with $m_{v} \leq 21$ is about 0.9 per sq arcmin at $90^{\circ}$ galactic latitude, and almost ten times greater at lower galactic latitudes. Therefore the PSF's used in these restorations were assumed without noise-contamination. A few applications carried out on both point-like (binary star) and extended objects are presented hereafter.

\subsection{Reconstructed images of binary star objects}

We have applied the reconstruction method to binary stars of different relative magnitude, and outlined effects of the SNR on both astrometric and photometric precision of the reconstruction. In the two examples presented here, we have chosen extreme magnitudes and magnitude differences. Note that we have considered 4 equidistant parallactic angles $\left(0^{\circ}, 45^{\circ}, 90^{\circ}\right.$ and $\left.135^{\circ}\right)$, and each star was located on a pixel of the $64 \times 64$ pixels array, which leads, with the sampling of 4 pixels per fringe adopted, to a 0 ". 10 field of view. The separation of the binaries were fixed to a value of 14.1 pixels, corresponding to 22.6 mas i.e. about 3 times the diffraction angular resolution limit, and the orientation angle to $45^{\circ}$. The relative photometry was computed by measuring the peak pixel values of the reconstructed sources since the sources were initially located at integer pixel locations.

In the first case, $\Delta m_{R}=1$ and $m_{R}=29$ for the main component. This leads to an average peak SNR of only 5.5 for the simulated interferograms. If we do not consider fringe pattern overlapping between main star and companion, the peak SNR for this latter is only 2.2 (see Fig. 1). Concerning the reconstructed object, the binary location is fully retrieved, while we will comment separately on the photometric accuracy.

In the second case, $\Delta m_{R}=2.5$ and $m_{R}=27.5$ for the main component. This leads to an average peak SNR of 11.3 for the simulated interferograms, and 1.1 for the companion. As in the first case, the reconstruction leads to a fully retrieved "astrometric" position of the original object. The relative magnitude, after 1000 iterations, of the reconstructed object is 3.1 , a good result when the difficulty of detecting this faint companion in the noise level is considered (see Fig. 2).

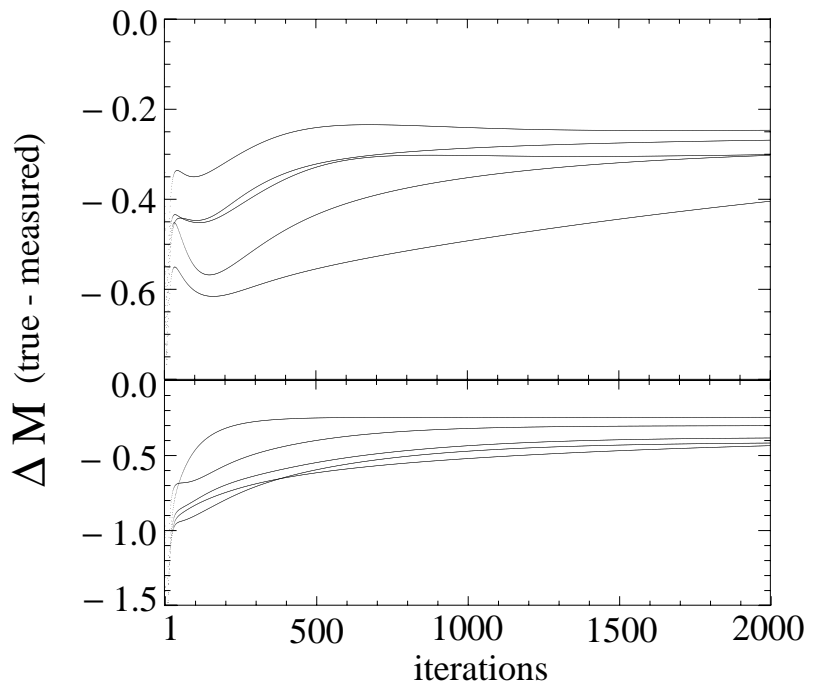

Fig. 3. Residuals of the magnitude difference for the $m_{R}=$ $29-30$ binary (top) and the $m_{R}=27.5-30$ binary (bottom) reconstructions as a function of the iteration number, and for 5 independent noise realizations

In both cases, we measured a magnitude difference larger than the true magnitude difference. We noticed however that this discrepancy was significantly reduced in tests with higher SNR. In Fig. 3, we show the variation of the photometric accuracy with iteration number. The residuals of the magnitude difference appear to remain stable after several hundred of iterations in both cases. Note also that, even though the algorithm convergence takes place essentially in $<10^{2}$ iterations (Fig. 4), the photometric accuracy seems to continue to improve, but this fact is only a consequence of performing photometry on the peak pixel. For the purpose of the present work, we were satisfied to verify this stability, and did not concern ourselves with a criterion to stop the iterations. In practical applications, the algorithm will be stopped according to the usual considerations on noise.

\subsection{Reconstructed image of an extended object}

We also tested the ability of the algorithm to reconstruct images of extended objects from LBT interferograms. The target used here is an image of the spiral galaxy NGC 1288 rebinned in a $128 \times 128$ pixels array that, with the sampling adopted, corresponds to a field of view of 0.20 in $R$-band. The image was apodized in order to avoid the effect of edge discontinuities in the restoration. In this example, the reconstruction was based on simulated observations at 6 equidistant parallactic angles $\left(0^{\circ}, 30^{\circ}, 60^{\circ}\right.$, $90^{\circ}, 120^{\circ}$ and $\left.150^{\circ}\right)$. With the $1000 \mathrm{~s}$ integration time assumed per parallactic angle, the total integration time is about 1.7 hours. The magnitude of the galaxy was set to $m_{R}=19$, which leads to approximately $210^{7}$ photons per 


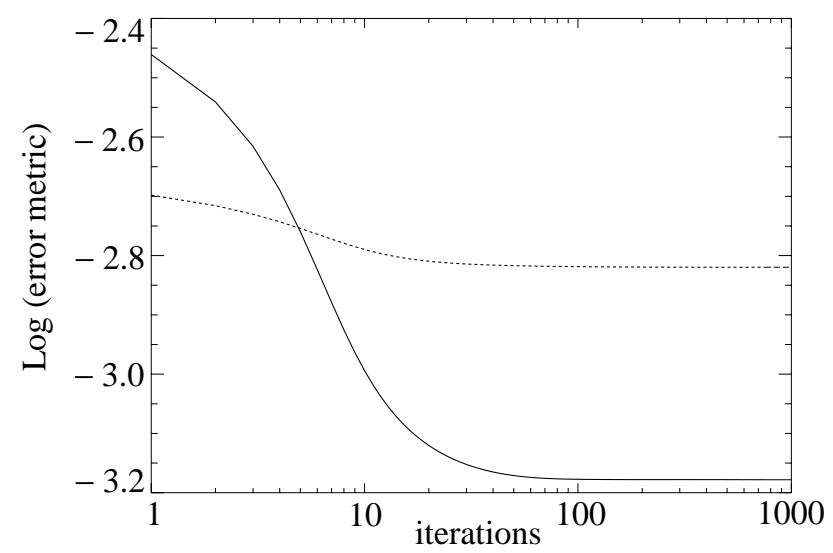

Fig. 4. Variation of the error metric with iterations number for the $m_{R}=29-30$ binary (solid line) and the $m_{R}=27.5-30$ binary (dashed). Error metric is defined as the sum, for all parallactic angles, of the Euclidian distance between the simulated interferogram and the convolution of the result with the PSF

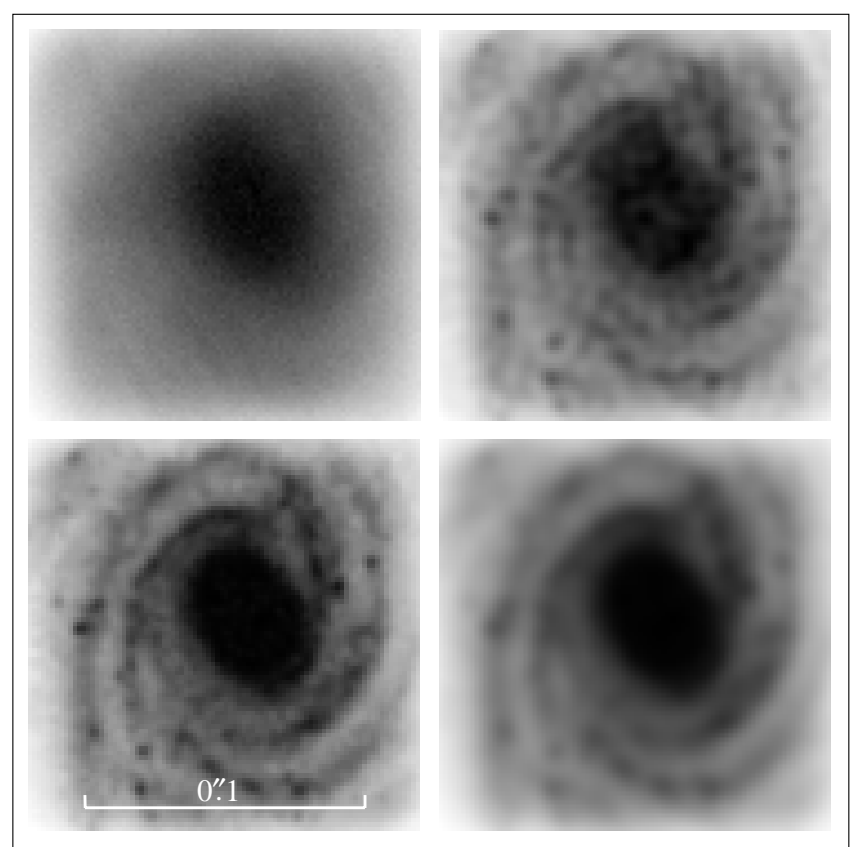

Fig. 5. Numerical simulation of interferometric imaging of an extended object with LBT. Top left: one of the observed interferograms of the $0{ }^{\prime \prime} 14$ field of view of a $m_{R}=19$ galaxy (parallactic angle $=30^{\circ}$ ), obtained after an integration time of $1000 \mathrm{~s}$. Bottom row shows the simulated target, unfiltered (left) and band-pass limited to a $22.65 \mathrm{~m}$ perfect circular aperture (right). Top right: result of the algorithm for 6 interferograms of $1000 \mathrm{~s}$ integration time each after 150 iterations

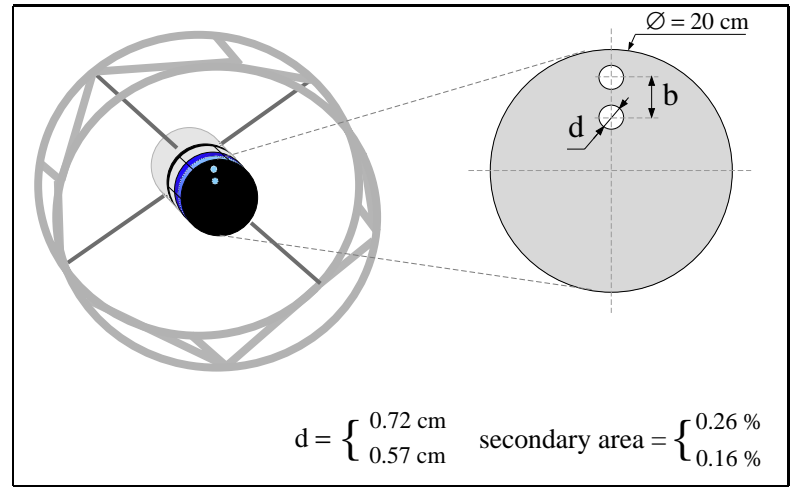

Fig. 6. Description of the interferometric experiment with the TIRGO telescope. Representation of the mask simulating the LBT pupil, and how it was mounted on the TIRGO secondary mirror. The ratio $b / d$, where $b$ is the center-to-center baseline and $d$ the diameter of each aperture, is equal to that of LBT, i.e. $b / d \sim 1.76$. The two values of $d$ used during the run are mentioned, as well as the resulting fraction of collecting area

Table 1. Observation conditions and resulting value of parameter $D / r_{0}$ ( $J$-band). $D$ is the projection of $d$ on the primary. Median seeing estimates of each night was provided by averaging 3 long exposure FWHM measures taken without the mask and spread over the night

\begin{tabular}{lllll}
\hline$d(\mathrm{~cm})$ & $D(\mathrm{~cm})$ & seeing $\left(^{\prime \prime}\right)$ & $r_{0}(\mathrm{~cm})$ & $D / r_{0}$ \\
\hline 0.72 & 5.4 & $4.0 \pm 0.5$ & $6.4 \pm 0.8$ & $0.84 \pm 0.11$ \\
0.57 & 4.3 & $3.5 \pm 0.5$ & $7.4 \pm 1.1$ & $0.58 \pm 0.08$ \\
\hline
\end{tabular}

long exposure image and a peak SNR of 80 . One can notice the sharper aspect of the reconstructed galaxy shown in Fig. 5 with respect to the theoretical diffraction-limited image. This is due to the behaviour of the algorithm in cases of high SNR. Also, a closer inspection shows that the nucleus does not appear as smooth as in the original picture. This is a consequence of the phenomenon of "noise amplification", which basically arises for such a maximumlikelihood deconvolution algorithm from the difference of converging rate between extended objects and point-like features (White 1994). For our aim, we did not concern ourselves with this problem, but different approaches concerning the solution of this drawback of the LR algorithm can be found in the literature (Lucy 1994; White 1994; Waniak 1997).

\section{Experiment at the TIRGO telescope}

In early 1998, we started an experiment at the $1.5 \mathrm{~m}$ TIRGO infrared telescope, with a mask simulating the pupil of the LBT telescope. The main idea was to record realistic LBT-like data by simulating the level of adaptive optics (AO) correction expected for LBT by the choice of the ratio $D / r_{0}$ (where $D$ is the diameter of one of the mask apertures projected on the primary and $r_{0}$ the Fried parameter), in order to investigate the properties of the atmospheric parameters and study the process of image formation and reconstruction (Fig. 6). 
An observing run, on the nights of 21 and 22 March 1998, permitted to collect a serie of measurements of one point-like and one binary star under two different values of the ratio $D / r_{0}$ (Table 1$)$. Actually, it can be noticed that the reduction in $D / r_{0}$ obtained with the use of a mask (i.e., by making $D$ smaller) is not equivalent to that expected from the use of adaptive optics on the real LBT (i.e., by making $r_{0}$ larger). In fact, in our case the mask holes produce a rescaling of the frequencies in the turbulence power spectrum. The effect of correction by adaptive optics, on the other hand, is not equal at all frequencies. But, for the aim of this experiment, these two corrections present enough similarities to be considered equal in first approximation. In order to simulate the rotation of the LBT-like pupil function, i.e. the aperture synthesis by earth rotation, we recorded object interferograms at four nearly equidistant mask orientation angles by rotating the secondary mirror. The data were recorded in a broad band $J$ filter at the $1.5 \mathrm{~m}$ TIRGO infrared telescope using the ARNICA camera (Lisi et al. 1996) which is equipped with a $256 \times 256$ pixels NICMOS 3 detector and presents a pixel size of 0.98 . During the run, we made use of the fast read-out mode of a $32 \times 32$ pixels sub-array, developed for lunar occultations, which allows typical integration times of around $20 \mathrm{~ms}$. A brief description of this mode can be found in Richichi et al. (1996). We present below a summary of some of the data reduction results.

\subsection{Measurement of atmospheric parameters}

A least-square PSF fitting program was developed and applied to our data in order to extract some atmospheric parameters of interest. It allowed us to retrieve the evolution with time of the fringe contrast, and of the random average optical path difference between the two apertures (that is the differential piston). This was done for the two values of the ratio $D / r_{0}$, on a 30 s period, and for an unique baseline orientation (Fig. 7), and we found consistent results in terms of average contrast and piston root-mean-square (rms) values. Indeed the case of major atmospheric degradation leads to a smaller average contrast: for $D / r_{0}=0.84$ and $D / r_{0}=0.57$ we found respectively an average contrast of 0.26 and 0.60 . Accordingly, we found a piston rms value of respectively 0.11 and 0.06 in unit wavelength.

In addition, combining several consecutive short exposure PSF interferograms, we studied the variation of contrast with integration time under the same two conditions of turbulence degradation (Fig. 8) and defined a coherence time $t_{\mathrm{c}}$ as the integration time which corresponds to a loss of a certain percentage of fringe contrast. We deduced the value of $t_{\mathrm{c}}$ with $10 \%$ loss for our experiment and attempted to extrapolate the result to the LBT case (Correia 1998).

\subsection{Application of the reconstruction technique to real data: $\gamma$ Leo}

The aperture size of the mask and the seeing conditions led, for this observation, to $D / r_{0}=0.84$. In the following, we used mainly a data set of $100 \mathrm{~ms}$ integration time exposures. This data set is composed of a total of 1200 interferograms of the binary $\gamma$ Leo (Algieba), a sequence of 300 interferograms for each of the four different mask orientation angles $\left(0^{\circ}, \simeq 46^{\circ}, \simeq 86^{\circ}, \simeq 126^{\circ}\right)$. The object is a binary star (ADS 7724) composed of a K1III main component and a G7III companion. According to the Hipparcos catalogue(ESA 1997) and to calibrated $V-J$ colors, the main component magnitude and the magnitude difference are respectively $m_{J}=0.21$ and $\Delta m_{J}=1.39$. The angular separation is $4 . " 58$, almost two times the diffraction limit of the simulated interferometer. In addition, 300 interferograms of an unresolved bright star (BS 5589, a M4.5III star with $m_{J} \simeq 0$ ) were recorded for the first mask orientation. These reference data are used for the system response (optics + atmosphere), the so-called PSF, in the deconvolution process.

In this run, the total collecting aera was limited by the fixed pixel size of the camera. For this reason, only $0.26 \%$ of the mirror area was used, leading for this integration time to a relatively poor signal-to-noise $(\mathrm{S} / \mathrm{N})$ ratio on the frames. Assuming frozen turbulence driven by the wind velocity $v$, the characteristic evolution time is of the order of $B / v$, where $B$ is the projection of $b$ on the primary i.e. here $B=9.5 \mathrm{~cm}$. Taking $v=10 \mathrm{~m} / \mathrm{s}$ leads to a typical evolution time of the order of $10 \mathrm{~ms}$. Consequently, the level of atmospheric degradation conditions (which is evaluated from the $D / r_{0}$ ratio to a $46 \%$ Strehl ratio) allowed us to assume that the blurring arising from high order atmospheric turbulence of evolution time scale inferior to $100 \mathrm{~ms}$ remains important. Note that, in order to minimise variations in seeing conditions during data acquisition, all frames of both binary and point-like stars have been recorded during consecutive periods of the night.

In the reconstruction, we used 200 interferograms of the binary star per orientation angle. A total of 800 interferogram patterns were pre-processed before applying the reconstruction method. Pre-processing consisted in subtracting the average sky background, a bilinear upsampling from $30 \times 30$ pixels to $90 \times 90$ pixels and then the extraction of a $64 \times 64$ pixels frame centered on the photocenter fringe pattern (see Fig. 9). This oversampling technique allowed a sub-pixel centering of each original $30 \times$ 30 pixels interferogram, i.e. to remove the atmospherically induced image motion.

Moreover, in order to obtain an accurate PSF estimate needed for the LR algorithm, a Shift-and-Add (SAA) process was computed over the whole PSF data set. Then we used the same pre-processing of the object interferograms to obtain the PSF estimate (see Fig. 9). Unfortunately, 

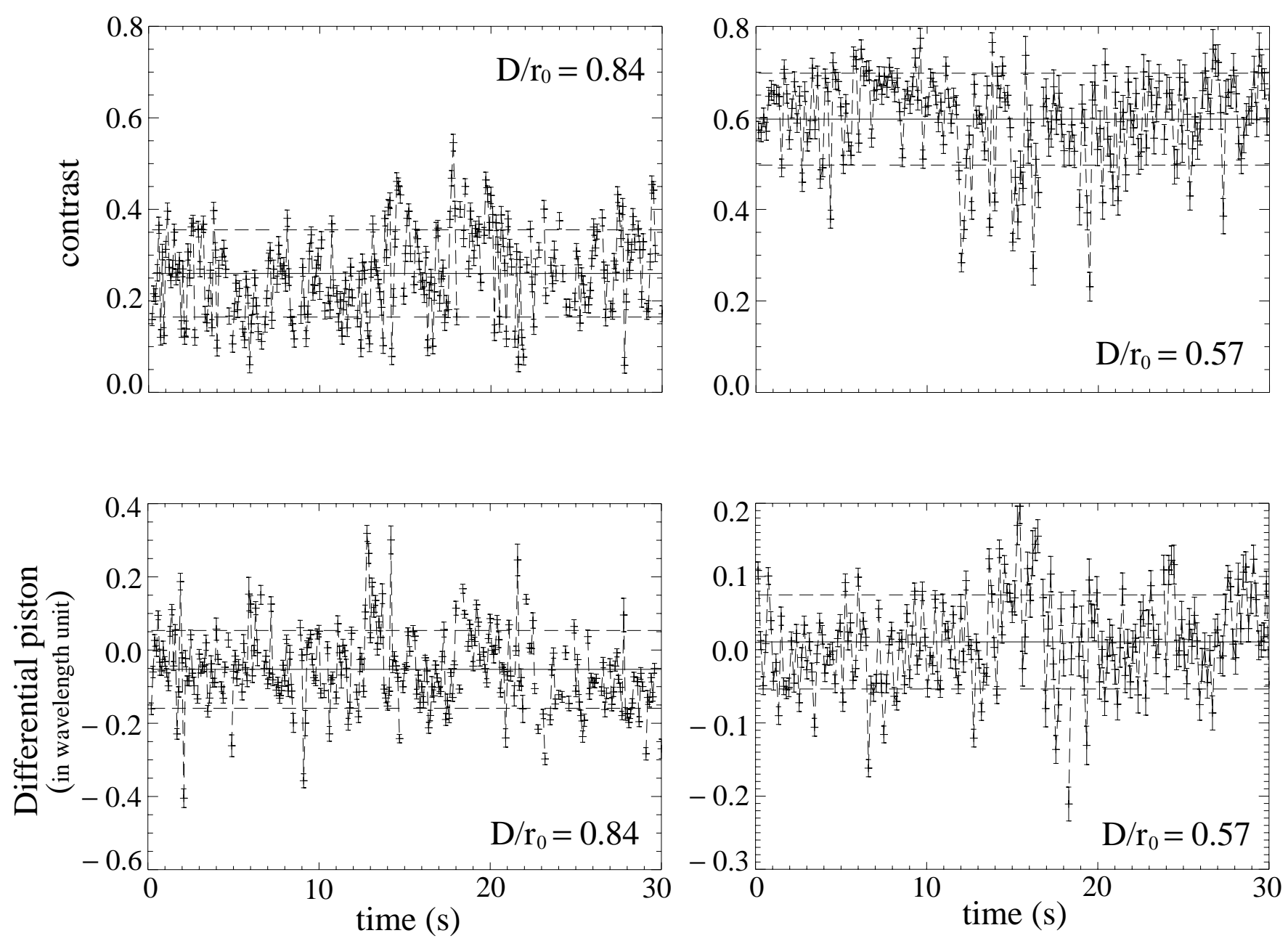

Fig. 7. Measure of atmospheric parameters obtained by fitting PSF data of the experiment. Evolution of fringe contrast (top row) and of differential piston (bottom row) in the data set for $D / r_{0}=0.84$ (integration time of $100 \mathrm{~ms}$ ) and $D / r_{0}=0.57$ (integration time of $150 \mathrm{~ms}$ ). The dashed lines represents rms intervals
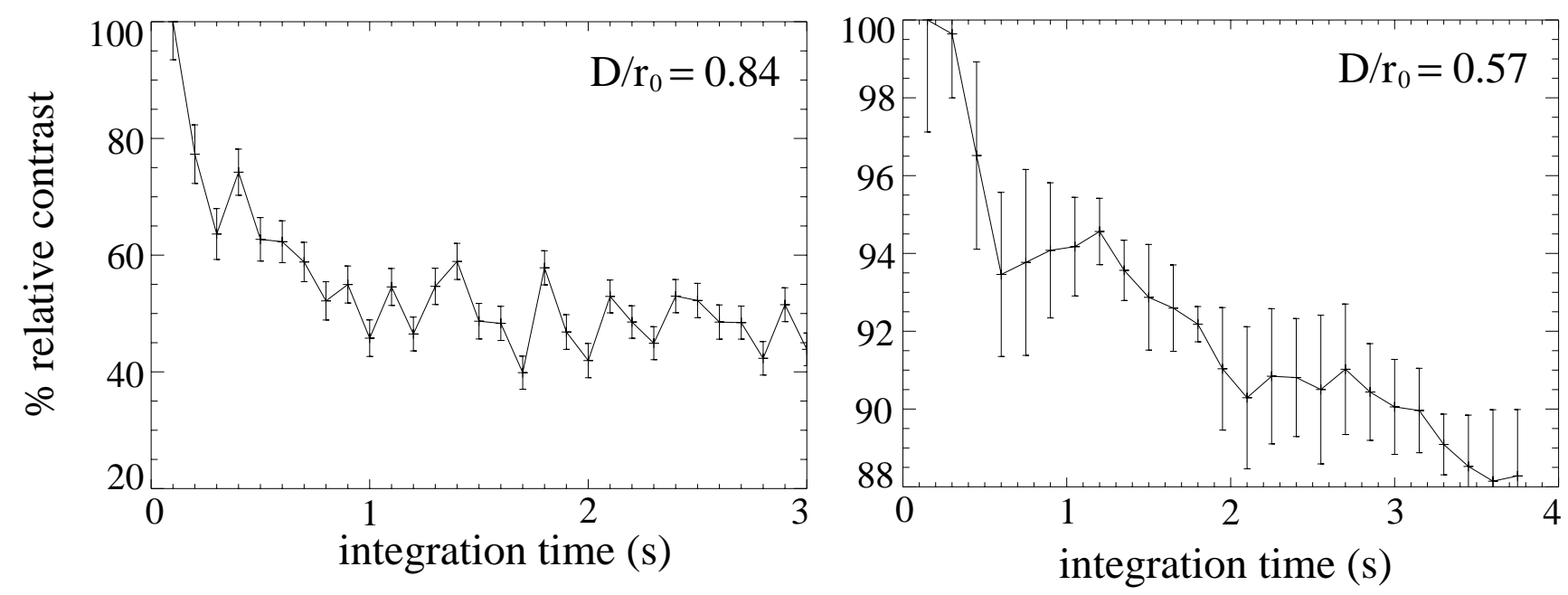

Fig. 8. Variation of relative fringe contrast with integration time, in relative value, obtained by coadding, for each integration time, several series of consecutive PSF interferograms 


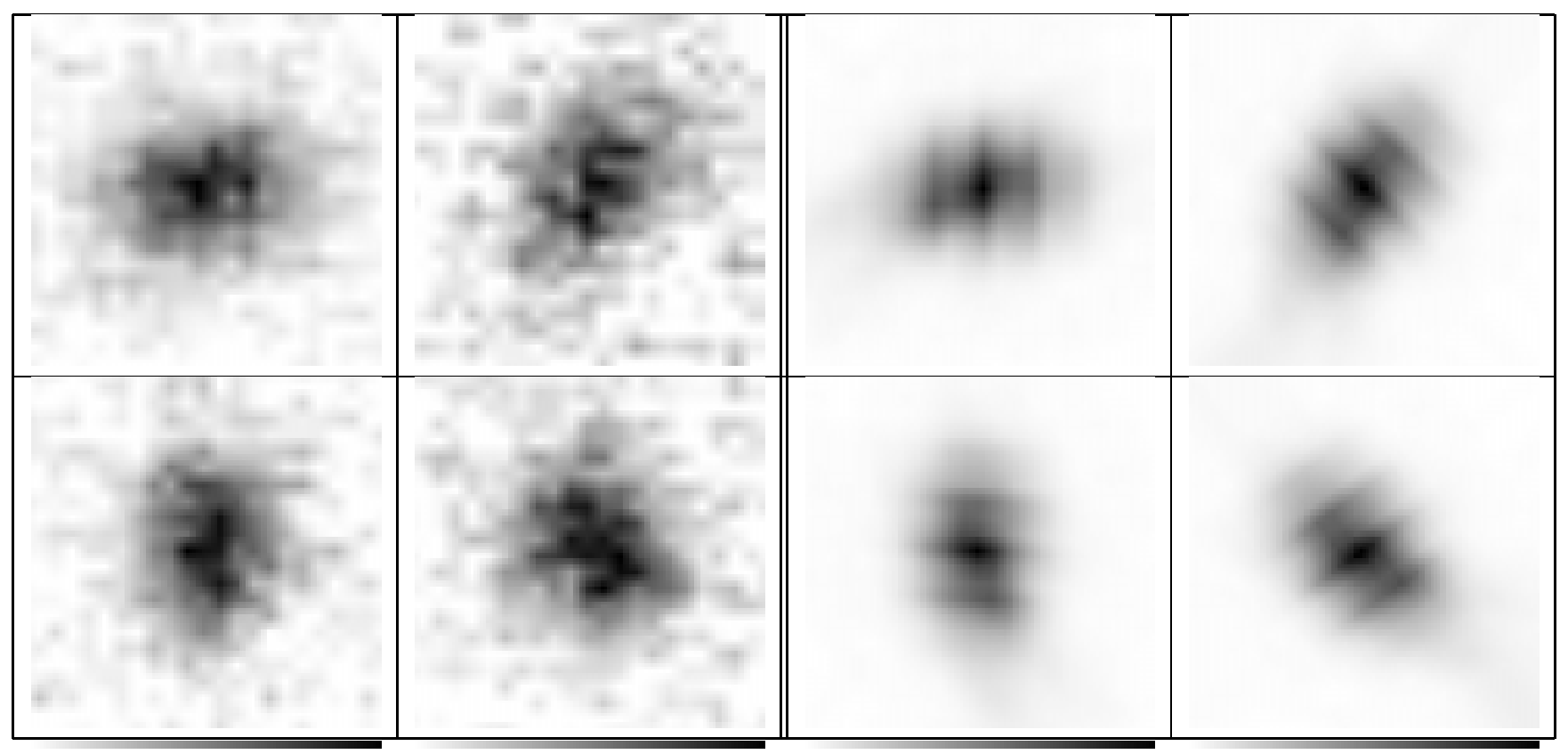

Fig. 9. Left panel: Set of the $64 \times 64$ pixels pre-processed interferograms patterns of the binary $\gamma$ Leo corresponding to each orientation angle. Each corresponds to an integration time of $100 \mathrm{~ms}\left(D / r_{0}=0.84, J\right.$-band $)$. Right panel: Corresponding set of SAA PSF estimates used for the deconvolution process. The pixel size is 0. .'98/3 $\sim 0^{\prime \prime} 33$, North is right, East is down (images are displayed on a linear scale)
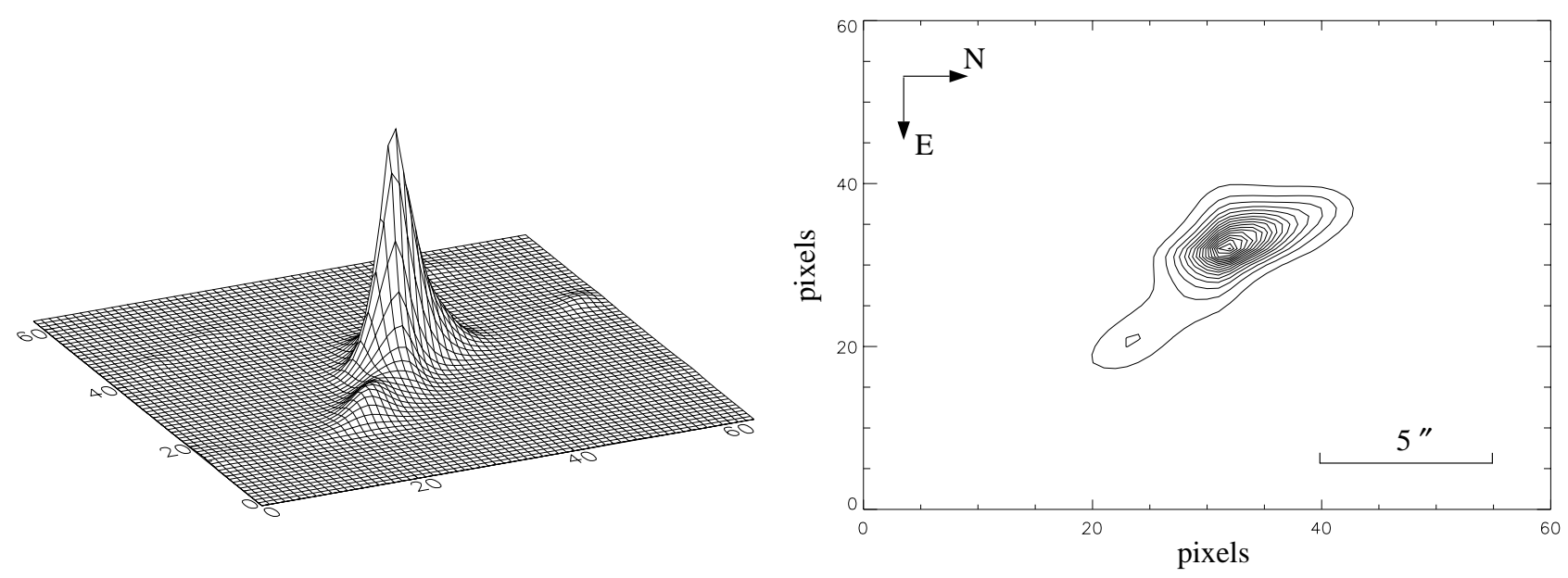

Fig. 10. Reconstructed image of the binary $\gamma$ Leo obtained using the Lucy-Richardson algorithm adapted for multiple deconvolution of simultaneous data frames (1000 iterations). The number of data frames per orientation angle used here is 200 , and each frame corresponds to $100 \mathrm{~ms}$ integration time. The pixel size is $0 . \prime 98 / 3 \sim 0^{\prime \prime} .33$. Contour levels are from $5 \%$ to $100 \%$ in steps of $5 \%$

the PSF was only recorded for the first orientation angle. The PSF corresponding to other orientation angles were obtained by computer rotation using a bilinear interpolation. Note that this rotation was performed on the oversampled frames, allowing to conserve more information in the PSF shape. However, it is unlikely that the responses of the system for each orientation angle are identical to the rotated responses. Therefore the rotated PSF's obtained in this way are only an approximation.
The companion is clearly visible in the reconstructed image of Fig. 10, in spite of numerous limitations in this data set. Indeed, in addition to the low $\mathrm{S} / \mathrm{N}$ ratio, and the approximation of the rotated PSF, the original data were also slightly under sampled $(\sim 1.8$ pixels per fringe FWHM ).

We further analysed the effect of the number of frames on the reduced data. Figures 11 and 12 show the evolution with this parameter of, respectively, the reconstructed object, and the profile of the reconstructed 


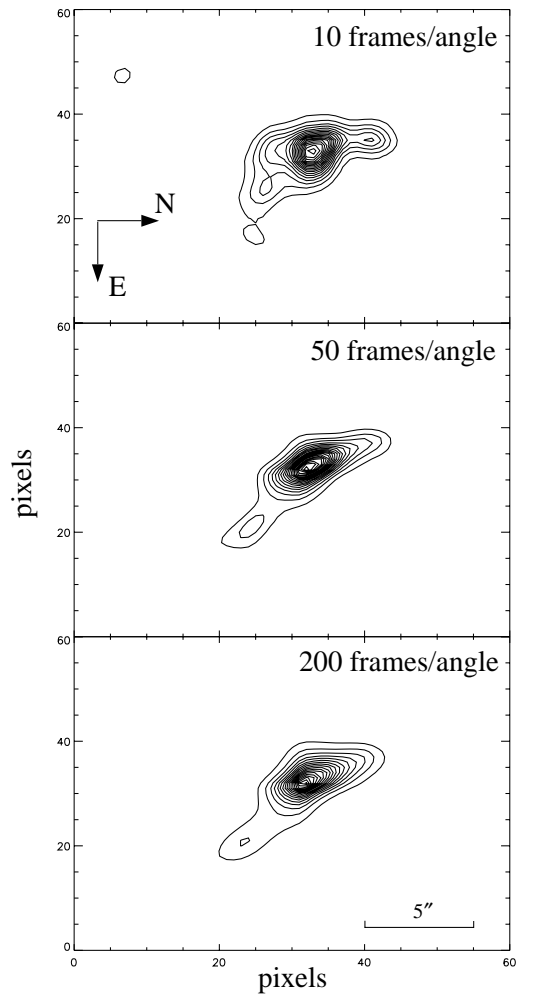

Fig. 11. Reconstruction of the binary $\gamma$ Leo according to the number of data frames per orientation angle processed, using the adapted LR algorithm (1000 iterations). The pixel size is 0 ". $98 / 3 \sim 0$ ". 33 . Contour levels are from $5 \%$ to $100 \%$ in steps of $5 \%$

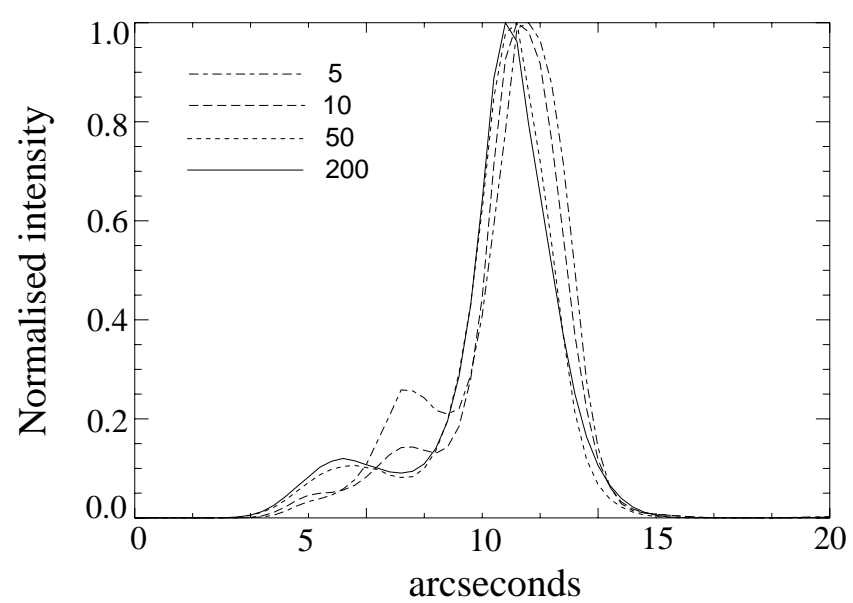

Fig. 12. Profile of the reconstructed binary along the direction of separation, recovered using different number of frames per angle

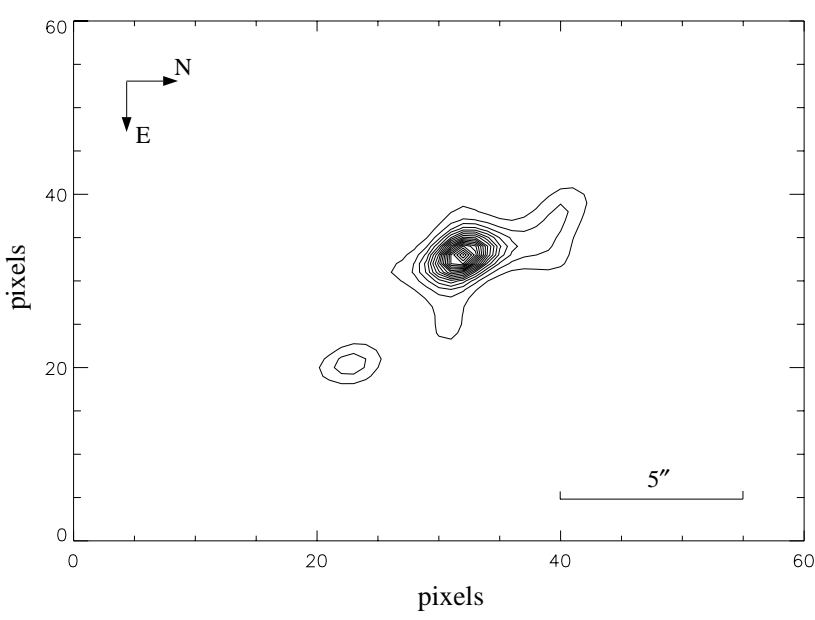

Fig. 13. Reconstructed image of the binary $\gamma$ Leo obtained deconvolving simultaneously 50 frames per orientation angle of $50 \mathrm{~ms}$ integration time each (1000 iterations). The pixel size is 0 .' $98 / 3 \sim 0$ ". 33 . Contour levels are from $5 \%$ to $100 \%$ in steps of $5 \%$

Table 2. Measured binary parameters retrieved from the deconvoluted images compared to the Hipparcos parameters of $\gamma$ Leo (HIP 50583). Measurements are both on $50 \mathrm{~ms}$ (50 frames/angle) and $100 \mathrm{~ms}$ (200 frames/angle) integration time deconvolution results and on data (PSF-fitting)

\begin{tabular}{lllll}
\hline & $\rho\left(^{\prime \prime}\right)$ & $\theta\left(^{\circ}\right)$ & \multicolumn{2}{c}{$\Delta m_{J}$} \\
\hline Hipparcos catalogue & 4.58 & 124.3 & \multicolumn{2}{c}{1.39} \\
& & & ap. phot. & PSF-fitting \\
Measured (100 ms) & 4.66 & 124.1 & $2.16-1.97$ & $1.58 \pm 0.33$ \\
Measured (50 ms) & 5.05 & 126.5 & $2.15-2.11$ & $1.56 \pm 0.11$ \\
\hline
\end{tabular}

binary along the direction of separation. It can be seen that the gain obtained in the resulting reconstruction using multiframes deconvolution is important for a small number of data frames, and tends to converge rapidly. The variation of the FWHM of the main component is consistent with the fact that deconvolving more frames simultaneously improves the quality of the reconstructed object, i.e. the sharpness of the reconstruction. In fact, for a number of 5, 10, 50 and 200 frames per angle used, the FWHM of the main component obtained is respectively 2 ". 47,2 ". 34,2 ". 31 and 2 "' 21 . It is interesting to note that we approached the theoretical diffraction limit for our mask of 1 "'73, but we were unable to reach it because of the limitations previously mentioned. It is however important to stress that this 2 ". 11 interferometric resolution does allow us to resolve $\gamma$ Leo, whereas the 5 ".83 resolution of only one aperture would not permit it. We noticed moreover that this simultaneous multiframes deconvolution leads to a better result than deconvolving simultaneously the sum of the frames for all baseline orientation. 
A data set composed of $50 \mathrm{~ms}$ integration time frames was reduced in the same way and the result obtained is presented in Fig. 13. This shorter integration time "freezes" better the atmospheric distorted fringe pattern and the result is a more detached binary image. Note that the spurious signal present on the main component sides probably came from a non perfect centering of each fringe patterns due to the poor $\mathrm{S} / \mathrm{N}$ ratio.

We tested the validity of these reconstructed images by performing the comparison of the retrieved binary parameter values with those of the Hipparcos catalogue (included in the CHARA catalogue, Hartkopf et al. 1998). Centroid calculations performed on $5 \times 5$ pixel boxes centered on each maximum intensity pixel of the two star brightness distributions led to the relative location of the binary. Two methods were used in order to measure the magnitude difference $\Delta m_{J}$. Firstly, relative aperture photometry was performed with aperture radii from 3 pixels to 7 pixels, and we deduced therefore a range of retrieved $\Delta m_{J}$. Secondly, we obtained photometry by PSF-fitting of the non-deconvolved frames, using the location retrieved by means of deconvolution as an input fixed parameter. This PSF-fitting is based on a least-square fitting routine identical to that used in Sect. 4.1 and was applied to a same number of coadded frames for each orientation angle. Results reported in Table 2 are an average over the four orientation angles, weighting with the $\chi^{2}$ in order to take into account the error fitting of the other free parameters. From the values reported in Table 2, one can see that we are able to retrieve both the position angle $\theta$ and the angular separation $\rho$ of $\gamma$ Leo, with a better accuracy in the $100 \mathrm{~ms}$ case. Concerning the relative magnitude retrieved by aperture photometry, $\Delta m_{J}$ is larger than the catalogue value by an amount of 0.77 to $0.58 \mathrm{mag}$ for respectively 3 to 7 pixels aperture radius and $100 \mathrm{~ms}$ integration time. This discrepancy is probably due to the poor $\mathrm{S} / \mathrm{N}$ ratio present in the recorded frames, and seems to be a common characteristic of most of the non-linear image restoration techniques when applied to strongly noise-contaminated data (Lindler et al. 1994; Christou et al. 1998). On the other hand, this interpretation is confirmed by the simulations performed in Sect. 3. Photometry measurements obtained by means of PSF-fitting lead to more accurate $\Delta m_{J}$ values and have the advantage to give error estimates.

\section{Conclusion}

We have presented a method for interferometric imaging with the Large Binocular Telescope. We have illustrated the capability of a Lucy-Richardson based deconvolution algorithm to reconstruct the object image from a set of LBT-like interferometric data. We outlined potential performances on simulated LBT data and robustness of the algorithm has been tested on real LBT-like data. It is of great importance, in order to achieve the reconstruction, to be able to obtain, from a reference star, a sufficiently good estimate of the PSF for each baseline position. The use of a Lucy-Richardson deconvolution algorithm is limited by the quality of the PSF calibration. The most favorable case would be to be able to record simultaneously object image and PSF. The LBT will provide a wide coherent field of view for interferometric imaging, thanks to innovative technological solutions (Salinari 1996). Therefore simultaneous measurements of both target and a bright reference star will be possible and, from this point of view, the algorithm presented here is of particular interest. The quality of the PSF calibration will be then limited by the difference of wavefront compensation achieved for these two objects. The future implementation of a AO correction in the simulation code will allow us to quantify this limitation.

Acknowledgements. We are indebted to F. Lisi and G. Marcucci for their help with the experimental set-up. We thank the director of the TIRGO observatory, Prof. G. Tofani, for the allocation of observing time. We are grateful to M. Bertero and P. Boccacci (University of Genova) for providing the initial version of the reconstruction algorithm, developed under contract number 16/97 funded by the Italian Consorzio Nazionale per l'Astronomia e l'Astrofisica. We wish also to thank P. Salinari, M. Carbillet and J.C. Christou for useful discussions, and A. Marconi and G.P. Tozzi for assistance during the observations.

\section{References}

Angel J.R.P., Hill J.M., Strittmatter P.A., Salinari P., Weigelt G., 1998, in: Astronomical Interferometry, Proc. SPIE 3352, Reasenberg R.D. (ed.), p. 881

Christou J.C., Hege E.K., Jefferies S., Cheselka M., 1998, in: Atmospheric Propagation, Adaptive Systems, and Lidar Techniques for Remote Sensing II, Proc. SPIE 3494, Davir A., Kohnle A., Schreiber U., Werner C. (eds.), p. 175

Correia S., 1998, Infrared interferometric imaging test for the LBT, DEA report, University of Nice Sophia Antipolis (available at http://www.arcetri.astro.it/ ${ }^{\sim}$ correia)

ESA, 1997, The Hipparcos catalogue, ESA SP-1200

Hartkopf W.I., McAlister H.A., Mason B.D., 1998, Third catalog of interferometric measurements of binary stars, CHARA (available at http://www.chara.gsu.edu/ DoubleStars/Speckle/intro.html)

Hege E.K., Angel J.R.P., Cheselka M., Lloyd-Hart M., 1995, in: Advanced Imaging Technologies and Commercial Applications, Proc. SPIE 2566, Clark N., Gonglewski J.D. (eds.), p. 144

Jefferies S.M., Christou J.C., 1993, ApJ 415, 862

Lindler D., Heap S., Holbrook J., et al., 1994, in: The Restoration of HST Images and Spectra II. Space Telescope Science Institute, Hanish R.J., White R.L. (eds.), p. 286

Lisi F., Baffa C., Biloti V., et al., 1996, PASP 108, 364

Lucy L.B., 1974, AJ 79, 745 
Lucy L.B., 1994, in The Restoration of HST Images and Spectra II. Space Telescope Science Institute, Hanish R.J., White R.L. (eds.), p. 79

Reinheimer T., Hofmann K.-H., Schöller M., Weigelt G., 1997, A\&AS 121, 191

Richardson W.H., 1972, J. Opt. Soc. Am. 62, 55

Richichi A., Baffa C., Calamai G., Lisi F., 1996, AJ 112, 6

Salinari P., 1996, in Optical Telescopes of today and Tomorrow,
Proc. SPIE 2871, Ardeberg A.L. (ed.), p. 564

Shepp L.A., Vardi Y., 1982, IEEE Trans. Med. Imaging, Vol. MI - 1, p. 113

Waniak W., 1997, A\&AS 124, 197

White R.L., 1994, in The Restoration of HST Images and Spectra II. Space Telescope Science Institute, Hanish R.J., White R.L. (eds.), p. 104 